\title{
Más allá del empleo: la centralidad del trabajo de cuidados en el curso de vida de las
}

\author{
Beyond employment: the centrality of care work in the life course \\ of migrant women
}

Paloma Moré Corral

Recibido: 01/02/2021

Aceptado: $17 / 03 / 2021$

\section{RESUMEN}

El trabajo de cuidados constituye un conjunto de actividades indispensables para el sostenimiento de la vida y del tejido social. A pesar de ello, se trata de un trabajo invisibilizado y escasamente reconocido social y económicamente. Este artículo articula el enfoque del curso de vida y la perspectiva interseccional con el objetivo de analizar las implicaciones del trabajo de cuidados, ya sea o no remunerado, sobre las distintas etapas del ciclo vital de una generación de mujeres migrantes. Para ello se ha utilizado una metodología de tipo cualitativo a través de 24 entrevistas en profundidad a mujeres de origen ecuatoriano que residen en la ciudad de Madrid y que trabajan o han trabajado en el sector de los cuidados a personas mayores. Las conclusiones apuntan a que el trabajo de cuidados tiende a lastrar las trayectorias de las mujeres migrantes hacia la escasez de recursos y la invisibilidad social: por un lado, porque sus trayectorias laborales en España se han centrado en el sector de los cuidados, uno de los más infravalorados y peor remunerados del mercado laboral; por otro lado, porque en distintas etapas del ciclo vital han tenido que hacer frente a dinámicas familiares que implicaban cuidados no remunerados a escala transnacional. El artículo muestra distintas tensiones que genera en estas mujeres un modelo de cuidados que se asienta sobre su trabajo pero que no es capaz de garantizar su derecho a cuidar y ser cuidadas.

Palabras clave: trabajo de cuidados, migraciones internacionales, curso de vida, género, migrantes.

\section{ABSTRACT}

Care work constitutes a set of essential activities for the sustainability of personal and social life. Despite this, it is an invisible work and is poorly recognized, both socially as well as economically. This article combines the life course approach and the intersectional perspective with the aim of analyzing the implications of care work, whether paid or unpaid, on the different stages of 
the life cycle of a generation of migrant women. The methodology if of a qualitative nature and consists in 24 in-depth interviews with women of Ecuadorian origin who live in the city of Madrid and who work or have worked in the sector of care for the elderly. The conclusions suggest that care work tends to weigh down the trajectories of migrant women towards scarcity of resources and social invisibility: on the one hand, because their work careers in Spain have focused on the care sector, one of the most undervalued and lowest paid in the labour market; on the other hand, because at different stages of the life cycle they have had to face family dynamics that implied unpaid care on a transnational scale. The article shows different tensions generated in these women by a care model that relies on their work but is not capable of ensuring their right to care and be cared for.

Keywords: care work, international migration, life course, gender, intersectionality. 


\section{INTRODUCCIÓN}

Este artículo analiza el curso de vida de una generación de mujeres ecuatorianas residentes en España a la luz de cómo el trabajo de cuidados, remunerado o no, ha ido conformando sus trayectorias en las distintas etapas del ciclo vital.

El contexto de esta investigación es la crisis de los cuidados (Pérez-Orozco, 2006) que tiene lugar en España a raíz de una serie de transformaciones sociales, entre las que destacamos el envejecimiento poblacional, la rápida incorporación de las mujeres al mercado laboral, y la escasa provisión de las políticas públicas para hacer frente a las crecientes necesidades de cuidados (Ibáñez y León, 2014: 110). Esta situación ha favorecido desde la década de 1990 el aumento de la mercantilización del trabajo de cuidados, particularmente en los hogares a través del empleo de hogar (Rodríguez, 2012; Martínez-Buján, 2014), pero también el crecimiento de un sector laboral profesionalizado (Recio, Moreno-Colom, Borràs y Torns, 2015) a través de servicios de atención domicilaria y centros residenciales para personas mayores (Moré, 2017). El crecimiento de estos sectores laborales coincide con la transformación de España en un país receptor de inmigración internacional (Cachón, 2009) y con el paso de un modelo de cuidados caracterizado por el "familismo impícito" (Leitner, 2003) a otro que ha sido señalado como el modelo "una inmigrante en la familia" (Bettio, Simonazzi y Villa, 2006).

A través de un estudio cualitativo en el que se realizaron entrevistas en profundidad a mujeres de origen ecuatoriano que residen en Madrid y que trabajan o han trabajado en el sector de los cuidados a personas mayores, este artículo utiliza el enfoque del curso de vida (Elder, 1991) y la perspectiva interseccional (Crenshaw, 1989) para analizar sus trayectorias de vida.

El objetivo del artículo es analizar el curso de vida de una generación de mujeres migrantes a partir de las experiencias relacionadas con el trabajo de cuidados, teniendo en cuenta que, tanto el trabajo remunerado como el que se realiza de manera gratuita y altruista en las redes familiares, es realizado mayoritariamente por mujeres. Así, de manera general nos preguntamos 
cuáles son los efectos o repercusiones que tiene este tipo de trabajo sobre las trayectorias de vida cuando se analizan desde una perspectiva diacrónica. Para ello, el análisis articula tres aspectos esenciales: las trayectorias laborales, los procesos migratorios y las posiciones en los núcleos familiares.

El artículo se organiza secuencialmente en apartados que analizan las diferentes etapas del ciclo vital, desde la juventud hasta la vejez. Puesto que tanto la edad como las etapas de la vida son socialmente construidas (Mortimer y Moen, 2016) estas se han delimitado en función de la vivencia de determinados procesos sociales y no de la edad biológica. La estructura del artículo es la siguiente: el primer apartado ofrece una aproximación al marco teórico de la investigación; el segundo presenta una contextualización de los flujos migratorios entre Ecuador y España haciendo especial hincapié en la inserción laboral de las mujeres en el sector de los cuidados; el tercero describe brevemente la metodología del estudio; el cuarto presenta los resultados exponiendo la centralidad del trabajo de cuidados en el curso de la vida a través de diferentes etapas de la trayectoria vital; el quinto apartado presenta unas reflexiones finales.

\section{ELEMENTOS DEL MARCO TEÓRICO: CURSO DE VIDA, INTERSECCIONALIDAD Y TRABAJO DE CUIDADOS}

El curso de la vida puede definirse como la secuencia de posiciones sociales que una persona adopta durante su trayectoria vital. Estas posiciones pueden estar relacionadas con transformaciones en el estado civil, los procesos de maternidad y paternidad, la residencia en un lugar determinado, o el trabajo, entre otros aspectos. Como enfoque analítico, el curso de vida busca interconectar las trayectorias individuales y familiares con los eventos sociales, culturales, económicos y políticos a nivel estructural (Elder, Kirkpatrick y Crosnoe, 2003). Para adoptar una visión de largo plazo, esta perspectiva se apoya en la noción de trayectoria entendida como "una línea de vida o carrera, un curso a lo largo de la vida, que puede variar y cambiar en dirección, grado y proporción" (Elder, 1991: 63). Las trayectorias no implican una secuencia específica de posiciones que los individuos deban seguir, sino que son flexibles y abarcan una amplia variedad de aspectos interrelacionados, como el trabajo, la escolaridad, la vida reproductiva o la migración (Blanco y Pachecho, 2003). 
Las distintas posiciones que adopta una persona dentro de su trayectoria se suelen denominar transiciones. Estas secuencias reflejan tanto los distintos períodos de la vida como los distintos estatus sociales por los que se transita, como pueden ser el matrimonio o el divorcio, el nacimiento de un hijo o una hija, la entrada o salida del mercado laboral o un proceso migratorio.

Desde la perspectiva del curso de vida se suele considerar que el trabajo remunerado es un estatus maestro (master status) que enmarca cada aspecto de la existencia social a lo largo del curso de vida, desde la juventud hasta la vejez (Moen y Roehling, 2005) y al mismo tiempo que el género es un elemento clave que afecta la naturaleza del trabajo y las carreras durante toda la biografía (Moen, 2016: 253). Sin embargo, sostenemos aquí que para captar la importancia fundamental del género en relación con el trabajo en el curso de vida es necesario trascender la noción de trabajo remunerado. Efectivamente, como ha puesto de manifiesto la literatura feminista, el trabajo doméstico y reproductivo amplía el concepto de trabajo entendido de manera restrictiva como sinónimo de empleo (Torns, 2008: 53; Carrasco, Borderías y Torns, 2011). En este sentido, más allá de la dicotomía entre el empleo (productivo) y el trabajo doméstico (reproductivo), la trayectoria laboral debe analizarse sociológicamente en un sentido amplio, considerando la variedad de formas en que las personas participan en el trabajo en la sociedad contemporánea (Parry, Taylor, Pettinger y Glucksmann, 2005: 4).

Además, nos parece fundamental que el enfoque del curso de vida se articule con la perspectiva interseccional (Crenshaw, 1989) para dar cuenta de cómo las trayectorias individuales se interconectan con las estructuras sociales dando lugar a situaciones específicas de desigualdad para distintos colectivos. En este sentido, la perspectiva interseccional pone de manifiesto que las relaciones de "raza", clase y género como estructuras de opresión diferentes pero interconectadas y que se construyen mutuamente (Collins, 1993: 26).

El enfoque del curso de vida define cinco principios básicos (Blanco y Pacheco, 2003) para la comprensión de las trayectorias vitales. Estos principios generales son herramientas útiles para analizar el trabajo de cuidados desde la perspectiva de la sostenibilidad de la vida y la perspectiva interseccional. El primero de estos principios establece que las trayectorias de desarrollo del 
individuo se extienden a lo largo de su vida (life-span development), haciendo referencia así a la necesidad de estudiar estos procesos a largo plazo con el fin de tener en cuenta las experiencias previas a la hora de comprender los comportamientos y decisiones de las personas. En este sentido si bien la división sexual del trabajo (Kergoat, 2000) es un elemento clave a la hora de analizar las trayectorias laborales femeninas, también debe tenerse en cuenta el contexto de relaciones sociales étnicas y de clase social (Anthias y Yuval-Davis, 1983; Lutz, 2019). En las trayectorias vitales no se pueden aislar las experiencias de género, clase, etnicidad y estatus migratorio, aunque dependiendo del contexto unos ejes de desigualdad van a resultar más significativos que otros. Además, las experiencias de desigualdad en función de las distintas posiciones sociales y sus interconexiones tienen efectos a largo plazo que se plasman en las etapas subsecuentes del ciclo vital.

El segundo principio se refiere a las limitaciones estructurales y en concreto a la influencia del contexto histórico y la ubicación espacial en la vida de los individuos o de las generaciones. En este sentido, las trayectorias aquí analizadas se enmarcan en el fenómeno global de división internacional del trabajo de cuidados que tiene lugar a partir de los años noventa a través de las migraciones internacionales de mujeres desde las regiones del Sur Global hacia regiones más ricas (Ehrenreich y Hochschild, 2002, Lutz, 2008; Anderson y Shutes, 2014; Amelina y Lutz, 2019). Desde el punto de vista de las sociedades de origen a menudo las mujeres emigran para hacer frente al empobrecimiento de su contexto de origen debido a las dinámicas ligadas a la globalización económica (Sassen, 2003). Desde el punto de vista de las sociedades de inmigración, distintos análisis con perspectiva interseccional (Glenn, 1992; Duffy, 2007) han dado cuenta de cómo el ámbito del trabajo reproductivo se transforma históricamente a través de la asignación de este trabajo a grupos sociales basados en el género, la etnia y la clase social y de los procesos de dominación entre distintos grupos.

El tercer principio ofrece un contrapunto al anterior al señalar la importancia de la capacidad de agencia (agency). Esta se refiere a que, dentro de un marco de limitaciones y oportunidades dado, las personas tienen también la capacidad de planificar y proyectar su propio curso de vida. En este sentido, las trayectorias 
de las mujeres migrantes muestran su capacidad para desarrollar sus propias estrategias de movilidad social a nivel personal e intergeneracional (Oso, 2016; Oso y Suárez-Grimalt, 2017). El cuarto principio es el del calendario (timming) que enfatiza la importancia del momento vital en el que ocurre un evento y la interacción entre el tiempo a nivel individual (edad), a nivel familiar (estatus en el ciclo familiar) y a nivel histórico (ciclos económicos, cambios sociales, etc.).

El último principio corresponde a la idea de que las vidas están vinculadas (linked lives), refiriéndose al hecho de que los cursos de vida son interdependientes, en particular en el contexto de los núcleos familiares. Este principio ilumina la centralidad de la interdependencia y la vulnerabilidad como elementos inherentes a la vida humana a lo largo del curso vital, así como la importancia de la reciprocidad asimétrica en la circulación de los cuidados (Baldassar y Merla, 2013). En este sentido, cuando definimos el trabajo de cuidado en sentido amplio, es decir no como un tipo de tarea concreta o centrado en un lugar específico como es el hogar (Anderson y Shutes, 2014), sino como "todos los elementos que se articulan en una red compleja de sostenimiento de la vida" (Fischer y Tronto, 1990: 40), es evidente que todas las personas necesitamos cuidados a lo largo de nuestra vida, aunque de distinto tipo e intensidad en función del proceso vital (Pérez-Orozco, 2014: 63). Por ello, como se ha señalado desde la economía feminista, el trabajo de cuidados es clave tanto a nivel macro, siendo un elemento fundamental para la reproducción social, como a nivel más personal, al ser parte constitutiva de la condición humana y determinar en gran parte la vida de las personas (Carrasco, 2016: 44).

La perspectiva del curso de vida puede contribuir a visibilizar la centralidad del cuidado en el sostenimiento de la vida al ser un trabajo que, ya sea remunerado o no, es socialmente imprescindible, a pesar de que se naturalice y se oculte tanto las tareas como quien las lleva a cabo (Carrasquer, 2013: 96). El trabajo de cuidados informal realizado de manera no remunerada y el trabajo de cuidados formal se intersectan muchas maneras a pesar de que las condiciones laborales y las trayectorias profesionales se organizan como si las personas no tuvieran obligaciones ajenas al trabajo remunerado (Moen y DePasquale, 2017: 2). La consecuencia es que los arreglos del cuidado son a 
menudo insatisfactorios e insuficientes (Pérez-Orozco, 2014: 65) tendiendo a crear situaciones de precariedad para quienes por su posición social no los pueden delegar en otras personas.

\section{MIGRACIONES INTERNACIONALES Y CRISIS DE CUIDADOS}

Con una cifra que supera ligeramente las 400.000 personas, Ecuador es el cuarto país de inmigración a España, tras Marruecos, Rumanía y Colombia, según datos del Instituto Nacional de Estadística (INE, 2020). Casi un tercio (31\%) de esta población se concentra en la Comunidad Autónoma de Madrid. En términos cuantitativos esta población ha experimentado varios cambios en las últimas décadas: a finales del Siglo XX era minoritaria, con unas 5.000 personas en 1998, experimentó un gran crecimiento en los primeros años del Siglo XXI hasta llegar en 2005 a las 487.239 personas, y después decrecer desde la crisis económica de 2008 hasta nuestros días (INE, 2020).

Entre las causas de esta migración suele señalarse el proceso de crisis financiera e inestabilidad política que Ecuador experimentó a finales de la década de 1990 y que llevó a la devaluación del Sucre, la moneda local, y a la posterior dolarización del país en 1999.

De manera simultánea, la economía española estuvo en expansión durante el periodo 1996-2007 lo que estimuló la llegada de población inmigrante para cubrir los puestos de trabajo más desprotegidos y peor remunerados del mercado laboral (Cachón, 2009). Como resultado, a principios de siglo, España se convirtió en un destino importante para los migrantes ecuatorianos, que antes emigraban a Estados Unidos. La emigración de población ecuatoriana a raíz de la crisis del 1999 se caracterizó por implicar a distintas clases sociales y a casi todas las regiones del país, pero concretamente a las mujeres (Herrera, 2005). En el año 1998 las mujeres eran el 58,7\% de la población nacida en Ecuador residiendo en España, proporción que en 2019 ha descendido hasta el $53,7 \%$.

De manera paralela a la transición migratoria en España se produce el fenómeno denominado como "crisis de los cuidados" (Pérez-Orozco, 2006) al 
resquebrajarse el modelo tradicional de división de roles de género ente el "hombre sustentador" y la "mujer ama de casa" que realizaba el trabajo de cuidados de manera invisibilizada y gratuita en los hogares. Este estatus quo se rompe cuando se normaliza el modelo de familia de "dos adultos sustentadores" pero sin conseguirse un notable incremento de la participación masculina en el trabajo doméstico, como muestran las encuestas de usos del tiempo (Ajenjo y García-Román, 2019). Esta problemática se agrava por las nuevas demandas ligadas a la atención de una población cada vez más envejecida, y sobre todo, por el escaso desarrollo de políticas públicas de provisión de bienestar que cubran esas nuevas demandas (Rodríguez-Cabrero et al, 2014). Como consecuencia, la familia sigue siendo el principal agente proveedor del trabajo de cuidados produciéndose además una mercantilización creciente a través de servicios en el mercado privado, principalmente a través del empleo de hogar (Martinez-Buján, 2014). En concreto, ha producido una revitalización del régimen de empleo de hogar interno (Ibid, 297) una modalidad de empleo que requiere que la trabajadora resida en el domicilio de sus empleadores y que se ha extendido particularmente en el caso del cuidado a personas mayores para asegurar una atención más flexible y personalizada incluso durante las noches. El sector laboral de los cuidados, especialmente aquellos dirigidos a la atención de personas mayores, se ha nutrido especialmente de población femenina inmigrante en estas décadas de expansión. Incluso, durante los periodos de crisis económica el empleo de hogar y en concreto el que está destinado al cuidado de personas ha resistido a la pérdida de empleo y la proporción de mujeres inmigrantes no ha dejado de aumentar (Díaz-Gorfinkiel y Martínez-Buján, 2018: 107-108).

\section{METODOLOGÍA}

Este artículo es parte de un proyecto de investigación postdoctoral que explora las trayectorias de vida y trabajo de las mujeres de origen ecuatoriano que han dedicado gran parte de su vida en España a cuidar de personas mayores en la ciudad de Madrid, ya sea como empleadas de hogar o como auxiliares de geriatría en servicios de ayuda a domicilio o en residencias de personas mayores. En concreto, se ha llevado a cabo una metodología cualitativa basada 
en la realización de 24 entrevistas en profundidad que abarcaron las trayectorias de vida de estas mujeres. Se ha elegido esta técnica porque la información biográfica recogida en entrevistas posee una dimensión interpretativa de la experiencia a partir de las representaciones asociadas a los acontecimientos vividos por la persona entrevistada (Alonso, 1998: 68). Debido a que los objetivos del estudio pretendían abordar la vivencia de las trabajadoras desde un punto de vista subjetivo, la construcción del "yo" a partir del discurso reflexivo de la entrevista constituía un punto central en esta investigación. Se trata de entrevistas largas, de entre una hora y dos horas y media de duración, realizadas en muchos casos en sus domicilios y también en lugares públicos como parques o cafeterías. El guion de entrevista proponía un recorrido por las distintas etapas del ciclo vital a través de preguntas dirigidas a abordar cómo en cada etapa se habían desarrollado las relaciones familiares y las actividades laborales. No obstante, las entrevistas fueron flexibles y si bien había un guion temático preestablecido, se dejó que la conversación fluyera libremente para que, siguiendo un hilo temporal, cada mujer conectara los temas según sus propias relaciones de sentido. Las entrevistas se realizaron entre los meses de octubre y diciembre de 2017. Después, con el conjunto de transcripciones se formó un corpus que fue codificado temáticamente a través del programa informático de análisis cualitativo MaxQDA. Esta codificación permitió ordenar la información estableciendo temáticas transversales del tipo "relaciones con el padre y con la madre", "estrategias de cuidados", "primer empleo en España", "proyecto migratorio", que aparecían en todas las entrevistas. Esto permitió realizar tanto un análisis individual de cada entrevista como un análisis transversal por contenidos específicos.

La muestra se obtuvo siguiendo criterios de representatividad sociológica y a través de la técnica de la bola de nieve'. En cuanto a las características de dicha muestra cabe señalar que la mayoría de las entrevistadas tienen entre 45 y 68 años, siendo solo tres de ellas más jóvenes, en torno a los 40 años. Estas mujeres forman parte de la misma generación de mujeres pioneras de las cadenas migratorias hacia España ya que todas, excepto tres que lo hicieron más tarde reagrupadas por algún familiar, salieron de Ecuador en el período 1993-2002 siendo las primeras en emigrar de sus familias (ver Anexo 7).

En anexo se adjunta un cuadro resumen con las principales características sociodemográficas de la muestra. 


\section{EL TRABAJO DE CUIDADOS EN EL CURSO DE VIDA}

\subsection{Las responsabilidades de cuidados antes de emigrar}

El origen de estas mujeres es diverso, pero predomina un perfil que proviene del medio rural y de familias numerosas en las que los padres han sido propietarios de pequeñas fincas, empleados agrícolas o comerciantes, y las madres han ejercido una gran diversidad de actividades domésticas para la familia como unidad de producción y consumo. Si bien algunas de las mujeres entrevistadas tienen estudios superiores, la mayoría no ha completado la enseñanza secundaria. En estos casos, sus trayectorias juveniles oscilan entre el trabajo asalariado para ayudar a la subsistencia de la unidad familiar y el trabajo de cuidados. Concretamente, el trabajo de cuidados en el marco familiar aparece como una explicación de la interrupción temprana de los estudios, como se puede ver en los siguientes ejemplos:

El primero es el de Melva, nacida en 1952 en Manabí, quien explica cómo su trayectoria vital quedó marcada por la transición de estudiante soltera a la de esposa y, sobre todo, madre, cuando deja de estudiar para poder criar a su hija.

Yo estudié el bachiller y primer año de enfermería. Me casé y dejé mi carrera. Pero, Bueno, me entregué totalmente yo a mi hija. (...) nos separamos pronto, pero bueno. ¡La vida es así! Continúa. Pues yo en la casa, pues yo ya mi cuarto donde yo tenía mi máquina [de coser] en la habitación de la niña (...) yo en la habitación la estaba viendo que estaba dormidita, pero yo estaba cosiendo. (Melva, 65 años, originaria de Manabi)

En el caso de Melva, como en tantos otros, la figura del padre está ausente de la crianza, y esto hace especialmente difícil poder continuar con un proyecto de formación y de trabajo fuera del hogar. Por ello, si bien se ve obligada a generar ingresos, tiene que hacerlo de manera compatible con los cuidados de una criatura, lo cual condiciona las oportunidades que tiene dentro del mercado laboral y recurre a realizar una actividad informal generadora de ingresos desde el hogar. Así, como explica, se dedica a coser "para una señora" desde casa y mientras vela el sueño de su hija. 
El segundo ejemplo es el de Cristina, nacida en 1955 en Ibarra, que se vio obligada a interrumpir sus estudios cuando a la edad de 16 años sus padres emigraron a Estados Unidos en la década de 1970 y ella y su hermana mayor tuvieron que asumir la responsabilidad de cuidar de cuatro hermanos menores. Esta situación fue sin duda un punto de inflexión en su trayectoria:

Yo fui solamente hasta la tercera de la secundaria que se llama en Ecuador. Porque pues ya mis padres en ese entonces ya... se fueron a Estados Unidos, y a partir de ahí ya tuvimos que nosotros cada uno los mayores tratar de buscarnos la vida. (...) Yo soy la quinta [de nueve hermanos], pero he sido la que también he tratado de ayudar. Yo quise... mis sueños se me quedaron un poco frustrados. Yo quise ser siempre doctora en medicina. (...) Entonces, claro, me vi obligada yo a trabajar. (Cristina, 62 años, nacida en Ibarra, Ecuador)

Este punto de inflexión motivó la precipitación de Cristina en el mercado laboral de manera abrupta y sin ninguna titulación, lo que tendrá consecuencias para el desarrollo de su trayectoria a largo plazo. Así, el proyecto migratorio de los padres de Cristina desencadena una serie de cambios en el núcleo familiar, que se dispersa y debe adaptarse a la nueva situación teniendo importantes consecuencias en el desarrollo posterior de la vida de esta mujer que asumió de manera temprana las responsabilidades del cuidado de sus hermanos pequeños. Si bien no todas las mujeres de esta muestra han tenido que hacer frente a similares responsabilidades en su juventud, muchas han asumido como madres la responsabilidad total de la crianza porque los hombres se desvinculan del trabajo de cuidados como un privilegio de su género. Estos ejemplos muestran cómo las trayectorias familiares están vinculadas (linked lives) y cómo el curso de vida está atravesado por las relaciones de género (Mortimer y Moen, 2016: 119).

\subsection{Un proyecto migratorio para sostener la vida}

Las decisiones en torno a los procesos migratorios no solo se toman en términos económicos, sino que implican estrategias familiares más amplias (Oso y Suárez-Grimalt, 2017). En el caso de las mujeres estas estrategias están marcadas por su posición de desventaja en las estructuras de género, que las hace más vulnerables a la falta de recursos y a distintos tipos de violencias. 
En este sentido retomamos el ejemplo de Melva, que ilustra cómo las mujeres jefas de hogar se insertan en "circuitos alternativos de supervivencia" (Sassen, 2003) a través de la emigración para hacer frente a la vulnerabilidad económica. Siendo madre separada tenía su vivienda hipotecada y día a día iba viendo como el coste de la vida aumentaba y no conseguía reducir su deuda. Impresionada por los relatos de prosperidad de los emigrantes a Estados Unidos, se decide a emprender su proyecto migratorio hacia España, donde le dijeron que había trabajo para mujeres, con la idea de buscar un futuro mejor para su hija. Para ello, tuvo que dejarla al cuidado de su abuela a la edad de nueve años y no la volvió a ver hasta que con 12 años viajó a España de vacaciones cuando "quiso quedarse, pero yo no la podía dejar porque yo estaba interna", hasta que finalmente se traslada a España con 16 años.

La maternidad transnacional (Parreñas, 2005) ha sido una pauta común para las mujeres de este estudio, ya que la mayoría tenían entre 30 y 45 años en el momento de emigrar y muchas eran madres que dejaron a sus criaturas con otras mujeres de su familia formando las denominadas "cadenas globales de cuidados" (Hochschild, 2001: 188). Este concepto evidencia el principio de las vidas vinculadas al mostrar que las experiencias de las mujeres envueltas en los movimientos migratorios para satisfacer la demanda en un mercado global de cuidados (Erenreich y Hochschild, 2002: 8), tenían un impacto sobre sus propios vínculos personales y familiares. En efecto, las estrategias de los hogares que implicaban mantener la esfera de la reproducción en el país de origen y a los miembros "productivos" en el país de destino (Oso, 2016), han sido muy frencuentes como forma de subsistencia, especialmente durante los primeros años del proyecto migratorio. En muchos casos esto ha implicado separarse de sus hijos e hijas cuando eran pequeños y volverlos a ver siendo adolescentes o jóvenes adultos. Este también es el caso de Silvia, que emigró en 2001 cuando su hija tenía seis años y se volvió a reencontrar con ella siete años después cuando regularizó su situación en España, transición que le permitió un horizonte de estabilidad al menos en términos de seguridad jurídica. En el caso de Milagros, originaria de Machala y nacida en 1972 y emigrada en 1996, dejó a su hijo de tres años con su madre y nunca lo llegó a reagrupar porque cuando por fin tuvo los medios materiales para hacerlo la separación entre abuela y nieto iba a ser 
demasiado dura. Todos estos casos confirman que cuando las familias migrantes permanecen separadas geográficamente los arreglos de cuidado tienden a enfocarse en las mujeres (Carling, Menjívar y Schmalzbauer, 2012: 193-195).

En otros casos, la emigración ha sido una forma de escapar, dando un giro a las trayectorias vitales, a la violencia machista, en un contexto donde el divorcio no siempre estaba bien visto. En este sentido, hay que señalar que la violencia machista ejercida por la pareja o por el padre ha sido un elemento que apareció espontáneamente en los relatos de una de cada cinco mujeres entrevistadas en este estudio.

Este es el caso de Marina, nacida en Quito en 1969, quien a través de la emigración buscó la forma de desvincular su trayectoria de la violencia física, psicológica, económica y sexual ejercida por su marido durante años. Se produjo un punto de inflexión en el momento en el que tuvo un aborto tras una paliza y decidió pedir ayuda económica y de cuidados a una de sus hermanas para poder viajar a España. Como ella afirma fue un acto desesperado: "Yo pedí ayuda a una hermana, le dije que me ayude a venir aquí porque jo me ayudaba o me mataba!". Cuando finalmente pudo emigrar tenía 31 años y era madre de cuatro hijas de entre doce y tres años. Hasta ese momento de su trayectoria su participación en el mercado laboral había sido mínima ya que se había dedicado a la crianza a tiempo completo y porque el control ejercido por su marido no le había permitido insertarse en ningún empleo. Con una mínima formación académica y experiencia laboral y un pasado marcado por la violencia más brutal, Marina llega a Madrid en 2001 habiéndose separado de su exmarido, pero también de sus cuatro hijas, que se quedan al cuidado de su madre y de su hermana. Las responsabilidades de cuidado al otro lado del océano son grandes, especialmente al saber que no puede contar con el padre de sus hijas. En ese momento se produce otro punto de inflexión en su trayectoria al morir repentinamente en un accidente la hermana que se había quedado con dos de sus hijas. Esto implicó que ella, desesperada, dejase el empleo de interna que tenía porque no le permitieron trasladarse a Madrid a reunirse con otros familiares. A partir de entonces, Marina explica que su rol de madre se complica pues sus hijas: "comenzaron a rodar de mano en mano", 
encontrándose sin un hogar estable y sin que ella, en situación irregular en España, pudiera reunirse con ellas ni en España ni en Ecuador. De nuevo la desigualdad de género se cierne sobre ella, esta vez en forma de culpabilidad y prejuicio sobre su rol de madre, pues asegura que ante esa situación de desamparo sus hijas la responsabilizaron por haber emigrado.

\section{3. ¡Búscate una señora para cuidar!}

A su llegada a España, las trayectorias de vida de las mujeres ecuatorianas que llegaron a Madrid entre 1993 y 2002 estuvieron condicionadas por su acceso al mercado laboral en el servicio doméstico y en particular en la modalidad de trabajadoras internas, por ser los empleos rechazados por las mujeres autóctonas debido a su incompatibilidad con la vida personal y familiar y las exigentes condiciones laborales (Colectivo Ioé, 2001). Las redes informales contribuyeron en gran medida a facilitar estos procesos (Colectivo Ioé, 2013) estableciendo vínculos entre hogares de ambos países. Los contactos personales se activan para reducir los riesgos y la incertidumbre en la búsqueda de empleo minimizando el tiempo entre la llegada a Madrid y el primer día de trabajo. El caso de Melva ilustra este proceso pues a su llegada a Madrid se dirigió al hotel que había reservado (para mostrar en el control policial del aeropuerto), pero antes siquiera de entrar en la habitación la recogió una mujer ecuatoriana, amiga de una conocida y que trabajaba como empleada de hogar, para llevarla a hacer una entrevista de trabajo. Ese mismo día, sin llegar a deshacer la maleta, se quedó a trabajar de empleada doméstica interna cuidando de una niña mientras lloraba al acordarse de su hija.

Por su parte, Marina relata cómo en sus primeras semanas en Madrid recorría las calles buscando empleo en hostelería sin éxito, hasta que siguió el consejo de una compatriota que le dijo: "Mira, Marina, tú a estos bares no vas a entrar [a trabajar] porque te piden papeles. ¡Búscate una casa para limpiar, para cuidar una persona mayor!" Y efectivamente, poco después, encontró trabajo como interna cuidando de una anciana. 
Experiencias como estas muestran hasta qué punto la inserción laboral de las mujeres ecuatorianas en España ha estado marcada por aspectos estructurales que implicaron una estrechez de horizontes más allá del empleo de hogar. Un sector que, en esos años y todavía incluso hoy, adolecía de una regulación laboral que fomenta la vulnerabilidad de las trabajadoras y se presta a situaciones de explotación (Diaz-Gorfinkiel y Martínez-Buján, 2018: 113) que exacerbaba las desigualdades de género, clase social y origen étnico a las que se enfrentaban estas trabajadoras en el mercado laboral español.

El servicio doméstico ha sido el principal primer empleo en España para las mujeres ecuatorianas (Colectivo Ioé y Fernández, 2010) y por ello tenían más facilidad que sus compatriotas masculinos para emplearse (Herrera, 2005: 287). Sin embargo, esta facilidad inicial no resulta tan ventajosa ante la realidad de los bajos salarios y las precarias condiciones laborales (Rodríguez, 2012). La inserción en este sector está relacionada con la estrategia migratoria pues en muchos casos entraron en España como turistas quedando después de tres meses en situación de irregularidad administrativa. Debido a este estatus irregular se veían forzadas a aceptar cualquier empleo y a evitar tener que hacer gasto en alojamiento y manutención (Colectivo Ioé, 2001: 40). Los primeros tiempos del proceso migratorio se caracterizan tanto por la reducción al máximo de los gastos, como por la estrategia de aprovechar todo el tiempo posible para trabajar, incluyendo los descansos, los días festivos, fines de semana y también las noches, para así compensar los bajos salarios. Esta estrategia se mantiene en muchos casos incluso cuando se ha logrado regularizar la situación migratoria, pues a pesar de ello los bajos salarios y la inestabilidad laboral persisten (Moré, 2017). Al cabo de los años de jornadas intensas y noches sin dormir, dejando el autocuidado en un segundo plano para trabajar más horas, los cuerpos de las trabajadoras sufrirán las consecuencias.

\subsection{Crianza y dificultades de conciliación}

En las trayectorias vitales de estas mujeres encontramos numerosas interconexiones entre el trabajo no remunerado de cuidados y el empleo. De esta manera, aunque la experiencia migratoria se plantease como un proyecto laboral, cuando las obligaciones de cuidados se cruzan en las trayectorias de estas mujeres, han tendido a anteponer las necesidades de atención 
relacionadas con la crianza a la inserción laboral. Así lo muestra Leonor, nacida en Quito en 1969 y emigrada a España en 2002, cuando explica las sucesivas interrupciones de su trayectoria laboral:

Me iba en la mañana y salía a las siete u ocho de la tarde, me parece... Era todo el día. Tampoco estuve mucho tiempo por el tema de mis hijos porque el papá los cargaba en el camión, es que no se podía, porque tenían cinco, cuatro y tres añitos. Entonces a mí me daba mucha pena dejarlos todo el día ahí, con el papá en el camión, no era vida para ellos. Entonces yo lo dejé.

Más adelante, volvió a encontrar un empleo y después de tres años volvió a encontrarse en una situación similar al darse cuenta de que necesitaba más tiempo para dedicarle a sus hijos:

Mis hijos ya comenzaban en la adolescencia y había que tenerles un poco más de... cuidado. Porque salía [de casa] a las seis de la mañana y llegaba a las cuatro o cinco de la tarde. (...) Para una mujer a veces es muy difícil compaginar un trabajo con los hijos. Porque yo aquí no tengo a nadie, decir, bueno, mi hermana, mi prima... me ayudan un poco, pero vamos... (Leonor, 48 años, Quito)

Las palabras de Leonor ilustran las enormes dificultades para compatibilizar la sostenibilidad de la vida con jornadas laborales de cuarenta horas o más, horarios atípicos, tiempos de desplazamientos en una gran ciudad. A esto se suma la falta de derechos laborales en el caso del empleo no declarado y los restringidos derechos que tienen las empleadas de hogar, como por ejemplo la ausencia de prestación por desempleo. Además, este ejemplo muestra la vigencia de la distribución tradicional de roles de género, no solo porque el trabajo del marido reporte unos ingresos más elevados, como suele suceder de manera estructural en el mercado laboral, sino también por la vigencia de la adecuación de las figuras femeninas, y en particular la materna, a la satisfacción de las necesidades de cuidado en los hogares.

El ejemplo siguiente, la historia de Marina ilustra cómo confluyen las dos situaciones descritas anteriormente: por un lado, la acumulación de empleos para conseguir un salario digno con el sobreesfuerzo que esto implica, particularmente notable en este caso al tratarse de una mujer que aún se 
encuentra en el puerperio; por otro lado, que tras un intento de arreglo de cuidados que implica emplear a una cuidadora y que el padre pase las noches con las criaturas recién nacidas, finalmente la madre asume el rol de cuidadora principal saliendo del mercado laboral y quedando por tanto sin ingresos y sin prestaciones sociales.

A los dos meses que tuvieron mis mellizos me fui a trabajar [cuidando de una anciana de 94 años]. Me conseguí una chica que me ayudaba, porque entraba a trabajar de siete a las 13.45 y ya me quedaba en casa [por las tardes con los niños]. Entonces, me dijeron: ¿usted sabe de alguna persona para la noche?" Le dije: “¡Yo mismo!” Entonces, yo hablé con mi marido él y le dije: “¿Qué te parece voy a entrar por la noche? Y así tengo dos sueldos". Y me dice: "Bien". Ganaba $1400 €$. (...) O sea, entraba a las diez de la noche y salía al otro día a las 13.45. Entonces yo recogía a los mellizos con la chica que les dejé, entonces yo ya me quedaba con ellos hasta que mi esposo venga y él dormía con ellos. Como yo no les daba pecho, era todo biberón... (...) ¡Un año me quedé! [en ese trabajo] Ya... la abuela pues se puso mala y yo ya me fui. Porque ya era mucho, era de domingo a domingo, y ya no podía más. No pasaba con mis hijos, mi esposo pasaba con los niños y me decía: “Ya, por favor, para. Mira que te has tirado un año allí". (Marina, 48 ans, Quito)

Además, el trabajo de cuidados en el hogar contribuye a difuminar la línea entre el trabajo productivo y reproductivo, en particular cuando, en ocasiones, se permite a las empleadas de hogar compaginar sus arreglos de cuidado con su empleo. Este fue el caso de Judith nacida en Loja en 1970 y llegada a Madrid en 1993, cuando sus empleadores le permitieron llevar a su bebé recién nacida al hogar donde trabajaba cuidando de una enferma de cáncer en fase terminal. Una situación similar encuentra Silvia cuando en el hogar donde trabaja como interna no solo cuida de una mujer anciana enferma de Alzheimer, sino también de su nieto recién nacido, que es el bebé de la hija que ella dejó de niña en Ecuador y que años más tarde fue a vivir con ella a Madrid.

En definitiva, el trabajo no remunerado de cuidados que implica la crianza condiciona los procesos migratorios femeninos moldeando dos tipos de estrategias: por un lado, la separación física y geográfica del trabajo reproductivo y productivo ejerciendo así la maternidad de manera 
transnacional; por otro lado, poniendo en práctica frágiles arreglos de cuidados para conseguir conciliar, de manera privada, individualizada y precaria, su trabajo remunerado con las obligaciones familiares. Como consecuencia de esta última estrategia, las trayectorias laborales se ponen en segundo plano añadiendo una mayor vulnerabilidad a la precaria inserción laboral "sexuada" y "racializada" en el sector de los cuidados a domicilio.

\subsection{Hijas que cuidan de sus mayores}

Baldassar y Merla (2013) enfatizan la circulación del cuidado siguiendo la norma de "reciprocidad generalizada" dentro de las familias. Esto implica que las personas ocupan diferentes puestos y roles en los arreglos de cuidados según su momento en el ciclo de vida (Clark, Glick y Bures, 2009). Así, cuando sus progenitores envejecen estas mujeres migrantes comienzan a preocuparse por la manera de afrontar sus necesidades de cuidados.

Este énfasis en la reciprocidad se ve reforzado por el hecho de que las políticas públicas de protección social en Ecuador son limitadas y la mayoría de los padres y madres de estas mujeres emigrantes no tienen pensiones de jubilación al haber trabajado siempre de manera no declarada. Puesto que los adultos mayores son un grupo "invisible" para las políticas sociales en Ecuador (Herrera, 2013: 85), el apoyo económico que ofrecen los descendientes, en particular los migrantes, es una fuente central de ingresos que permite a las personas mayores acceder a la atención médica privada.

Teniendo en cuenta el alto costo de la atención sanitaria y social en Ecuador, muchas mujeres migrantes explicaron que hubieran querido traer con ellas a sus padres y madres para que pudiesen acceder a la sanidad pública (Skornia, 2015: 58). Sin embargo, este proyecto se hacía difícil por diversos factores, entre ellos la dificultad para cumplir los criterios para el proceso de reagrupación familiar, como por ejemplo la falta de viviendas adecuadas y de recursos económicos para mantenerles, o también el hecho de que no quisieran abandonar su entorno (Diaz-Gorfinkiel y Escrivá, 2012: 140). 
Se plantea así la situación de proporcionar cuidados desde la distancia, mientras se vive y trabaja cuidando de personas mayores en España. Tal es el caso de Mireia, de 39 años y originaria de Loja, que fue reagrupada por su hermana mayor en 2007 y trabaja cuidando de una mujer de 93 años:

[Enviar dinero] es que lo hacemos desde que estamos aquí. Mi hermana desde que vino acá es lo único que ha hecho. Eh... ayudar lo que más se ha podido. Y yo también, desde que he venido aquí es dedicarnos totalmente a la alimentación y medicina de nuestros padres (...) entonces pues, como le digo, tengo mi [otra] hermana que se dedica a cuidarlos y nosotras mandamos el dinero para que se los vean y todo eso.

(...) decir que nosotras les vamos a ir cuidar es difícil (...) la única manera de tenderles una mano a mis padres es estando aquí. (Mireia, 39 años, Loja)

Los cuidados de larga duración a personas mayores pueden requerir una presencia constante y un intenso trabajo material y las familias transnacionales deben organizar estrategias de cuidados complejas para hacer frente a esta situación. Estas estrategias son diversas, pero tienen la pauta común de que el género es el eje estructurador al definir diferentes grados de implicación y obligación para hombres y mujeres (Carling et al., 2012: 193-196). Cuando la persona cuidadora principal es una mujer, suele convivir con la persona mayor y realizar tareas de cuidado personal, como asear, dar de comer, preparar la comida, etc., mientras que cuando se señala que hay un hombre como cuidador principal, las tareas son típicamente masculinas, como conducir para realizar desplazamientos o hacer visitas puntuales al domicilio.

Por otro lado, los familiares migrantes se encargan de generar los recursos económicos para mantener los cuidados, desde la atención médica, hasta los gastos de manutención y vivienda. Como señala Boccagni (2011: 324), la protección social en Ecuador radica más en la acción espontánea de los migrantes que en los programas de gobierno social. Así, las mujeres migrantes consolidan, a través de los cuidados remunerados, su posición de proveedoras económicas para sus familiares en sentido amplio. En algunos casos, esto les permite tener recursos para comprar trabajo de cuidado en el mercado a través de empleadas de hogar en Ecuador. 
En otros casos, la doble nacionalidad ${ }^{2}$ les permite regresar a Ecuador durante un período más o menos prolongado de tiempo, generalmente aprovechando las vacaciones o los periodos en desempleo, para cuidar de un progenitor que se encuentra en una situación de dependencia o enfermedad grave. Estos suelen ser periodos cortos y de gran intensidad, caracterizados por la entrega desmedida al cuidado del ser querido y también por la urgencia del regreso, motivado no solo por las obligaciones laborales sino también por las responsabilidades en el cuidado de sus descendientes en España.

\subsection{Mujeres hacia la jubilación y el envejecimiento}

Los procesos de envejecimiento de las mujeres inmigrantes que trabajan en el sector de los cuidados están condicionados por años de trabajo largo e intenso, muchas veces de repetidos esfuerzos físicos y en condiciones precarias (Iglesias, 2010; Moré, 2017: 139, 323). Además, para quienes trabajan a domicilio y particularmente para las internas, la sensación de estar encerradas en el universo de sus empleadores y la falta de relaciones sociales con el mundo exterior son dificultades igualmente importantes (Moré, 2018: 7). Para quienes cuidan de personas mayores, el contacto cercano y continuo con una persona altamente dependiente con un deterioro cognitivo significativo puede contribuir a deteriorar su situación emocional (Moré, 2019: 20). En definitiva, el esfuerzo físico y el desgaste psicológico forman parte del trabajo diario y sus consecuencias son especialmente visibles en la vejez. Como explica Cristina, el trabajo de cuidados a personas mayores a domicilio implica un desgaste físico importante:

Cuando he cargado y levantado a las personas mayores. Que yo he trabajado mucho con personas mayores. Y siempre ha sido levantarles... Te vas acabando ahí. Sí o sí te vas acabando. (...) Yo sí me he matado en cambio porque he dado todo de mi. He dado todo de mi al cuidado, a pero, claro, también he sido tonta, no me he cuidado. (Cristina, 62 años, Imbabura).

Además, hay que tener en cuenta que estas mujeres suelen tener jornadas laborales muy intensas, con muy poco tiempo para descansar. En el caso las

\footnotetext{
2 A partir de los dos años de residencia formal en España, la población ecuatoriana, entre otras, puede beneficiarse de una excepción en la ley de naturalización e iniciar un proceso de solicitud de ciudadanía española (Finotelli y La Barbera: 2013). Como resultado, según el Instituto Nacional de Estadística, en 2019, el 70\% de la población ecuatoriana residente en España tiene nacionalidad española, el 73,2\% de quienes viven en Madrid. Las 24 mujeres participantes en este estudio tenían la doble nacionalidad.
} 
empleadas domésticas internas, pero también de las auxiliares del servicio de ayuda a domicilio o de quienes trabajan en residencias. A menudo quienes trabajan en estos empleos más profesionalizados es compensar los bajos salarios haciendo horas extras o trabajos no declarados de limpieza y cuidados por horas o por las noches, a domicilio o incluso cuidando de personas enfermas en hospitales. A esto se suman las horas de trabajo no remuneradas dedicadas a realizar las tareas domésticas en el hogar, así como al cuidado otros familiares, como se puede ver en el caso de Silvia:

A mi pues yo veo que [no me cuida] nadie. [Se ríe] Es que yo no sé, no... La verdad que sí que digo yo, yo he echado una mano a mi hija, ¿no? [En ese momento esta con su nieto recién nacido en brazos calmándole el llanto] Pongamos, aquí estoy echando una mano, mi trabajo, pero no sé, o será que no entiendo, pero muchas de las veces sí que tengo un agobio de mucho trabajo. Porque la verdad a veces mi esposo no es de los que dicen: "Te voy a ayudar a lavar este plato o me has servido y te voy a recoger." ¡Siempre son servidos todos! ¿Y yo qué? ¿Quién me ayuda? ¡Nadie! (Silvia, 52 años, Ibarra)

De cara al futuro, algunas afrontan la edad de jubilación con la certeza de poder recibir una pensión contributiva, aunque la cuantía no sea muy alta ya que las empleadas de hogar tienen unas pensiones especialmente bajas. Sin embargo, otras no llegan a cumplir el requisito mínimo de 15 años de cotización a la Seguridad Social para tener derecho a una pensión contributiva, debido al trabajo no declarado y también a las interrupciones en sus trayectorias laborales para dedicarse al trabajo no remunerado. Así, en algunos casos se encuentran con que en la vejez se plantean seguir trabajando, de manera informal, para compensar los bajos recursos que reciben a través de pensiones no contributivas y de ayudas sociales.

\section{REFLEXIONES FINALES}

El trabajo de cuidados tiende a lastrar las trayectorias femeninas hacia la escasez de recursos y la invisibilidad social puesto que se trata de un trabajo que a pesar de ser esencial adolece una falta de reconocimiento económico y social que favorece la naturalización y la invisibilidad la actividad y de quien la realiza. En el caso de las mujeres que han participado en este estudio esto se ve 
acrecentado por dos motivos ligados a la migración: por un lado, porque sus trayectorias laborales en España se han centrado en el sector de los cuidados, uno de los más infravalorados y peor remunerados del mercado laboral; por otro lado, porque en distintas etapas del ciclo vital han tenido que hacer frente a dinámicas familiares que implicaban cuidados no remunerados a escala transnacional.

A través de las trayectorias de estas mujeres se visibiliza el impacto que las desigualdades de género, de clase social y ligadas a su procedencia étnica y migratoria, tienen a largo plazo y en las distintas etapas del curso vital.

El enfoque del curso de vida permite visibilizar la importancia de las trayectorias a largo plazo y cómo el contexto histórico en el que se enmarcan determinadas transiciones, en este caso la emigración de Ecuador a España en los años de la crisis en Ecuador y la crisis de los cuidados en España, contribuye a trazar un perfil en el curso de vida de personas que no pertenecen a la misma cohorte. Este enfoque permite también iluminar la importancia de cómo los factores estructurales tienen un peso en las trayectorias sin olvidar que existe un espacio para ejercer una "agencia constreñida" (Moen, 2016: 251) que permite a las trabajadoras elaborar sus popias estrategias migratorias, laborales y de cuidados. Además, puesto que los cuidados implican necesariamente el mantenimiento de vínculos el enfoque del curso de vida resulta atractivo al llamar la atención sobre cómo las trayectorias entrelazan a lo largo del curso vital.

A través de las trayectorias de las mujeres migrantes que se encuentran vinculadas a dos Estados de bienestar familistas, España y Ecuador, los cuales fundamentan el trabajo de cuidados en las familias y dentro de ellas, en las mujeres, este artículo muestra las carencias de un modelo de bienestar totalmente insuficiente e injusto que se fundamenta en desigualdades estructurales de género, clase social y etnia a nivel global. Ante esta situación que moviliza a las mujeres como garantes del bienestar pero que no satisface sus propios derechos a cuidar y ser cuidadas, estas mujeres se enfrentan una serie de contradicciones que generan malestar: 
En primer lugar, como mujeres inmigrantes se les restringen las oportunidades laborales más allá del sector de los cuidados a las personas, pero al mismo tiempo se les niega o dificulta en extremo su derecho a cuidar de sus familiares y a disfrutar de la vida en familia. En segundo lugar, cuando deciden no renunciar a la vida en familia, y especialmente a la convivencia con sus criaturas, tienen que poner en segundo plano su participación en el mercado laboral debido a las dificultades para compatibilizar el empleo con los cuidados no remunerados ante la escasa corresponsabilidad (tanto a nivel de instituciones como familiar). En tercer lugar, debido a la escasa protección social para las personas mayores en Ecuador, los familiares migrantes se convierten en un recurso fundamental para el bienestar, así, en el caso de las mujeres, desempeñan el rol de proveedoras económicas y no de cuidadoras materiales, que sería su rol de género tradicional. Sin embargo, cuando se dan las circunstancias propicias para pasar un tiempo cuidando de un familiar mayor dependiente, la entrega es muy intensa, como si se tratase de compensar la desviación del rol tradiciona. En cuarto lugar, puesto que los cuidados son universales y esenciales, estas mujeres acceden fácilmente al empleo y lo pueden mantener durante las crisis, constituyéndose como un recurso valioso para el bienestar de sus familias, pero estos empleos les reportan escasos derechos de protección social para el momento en que no puedan ser empleables. Esto se hace especialmente visible en la vejez pues las pensiones a las que acceden son exiguas. Por último, el trabajo de cuidados es física y psicológicamente exigente y tiene consecuencias para el bienestar personal, especialmente negativas cuando se realiza en un entorno marcado por las malas condiciones laborales (intensificación del trabajo) y materiales (falta de material adaptado). Por ello, las trabajadoras de los cuidados acumulan en sus cuerpos fatigas, cansancios, malas posturas y mucho desgaste, que se hace patente en la vejez, precisamente en el momento en que sus recursos económicos no les permiten dejar de trabajar o acceder a comprar cuidados en el mercado privado y hace que dependan de las escasas ayudas públicas y de las redes familiares. 
Más allá del empleo: la centralidad del trabajo de cuidados en el curso de vida de las mujeres migrantes

\section{ANEXO}

\begin{tabular}{|c|c|c|c|c|c|c|}
\hline & $\begin{array}{c}\text { Nombre } \\
\text { (pseudónimo) }\end{array}$ & Edad & $\begin{array}{l}\text { Lugar de } \\
\text { origen }\end{array}$ & $\begin{array}{l}\text { Año de } \\
\text { inicio } \\
\text { residencia } \\
\text { en España }\end{array}$ & $\begin{array}{c}\text { Situación laboral en el momento de la } \\
\text { entrevista }\end{array}$ & Situación familiar \\
\hline 1 & Melva & 65 & Manabí & 1993 & $\begin{array}{l}\text { Limpiadora en oficinas. Percibe prestación } \\
\text { por Incapacidad Temporal (IT). En pocos } \\
\text { meses se jubila. }\end{array}$ & $\begin{array}{l}\text { Divorciada, tiene una hija y dos nietos/as que viven } \\
\text { en Barcelona. Vive sola. }\end{array}$ \\
\hline 2 & Gloria & 55 & Machachi & 2006 & $\begin{array}{l}\text { Trabajadora del Servicio de Ayuda a } \\
\text { Domicilio del Ayuntamiento de Madrid. }\end{array}$ & $\begin{array}{l}\text { Casada, tiene dos hijas adultas que viven en el hogar } \\
\text { familiar. }\end{array}$ \\
\hline 3 & Silvia & 52 & Ibarra & 2000 & $\begin{array}{l}\text { Empleada de hogar interna que cuida a } \\
\text { una persona dependiente. }\end{array}$ & $\begin{array}{l}\text { Casada, tiene un hijo, una hija y un nieto. Convive } \\
\text { con su marido, hija y nieto en el piso de su } \\
\text { empleadora (es interna). }\end{array}$ \\
\hline 4 & Cristina & 62 & Imbabura & 2000 & $\begin{array}{l}\text { Limpiadora en oficinas y empleada de } \\
\text { hogar por horas. Percibe prestación por } \\
\text { Incapacidad Temporal (IT) }\end{array}$ & $\begin{array}{l}\text { Divorciada, tiene un hija y dos nietos/as que viven en } \\
\text { Italia. Vive sola. }\end{array}$ \\
\hline 5 & Inés & 53 & Quito & 2001 & Desempleada. & $\begin{array}{l}\text { Divorciada, tiene un hijo y una hija adultos. Convive } \\
\text { con su hijo. }\end{array}$ \\
\hline 6 & Marina & 48 & Quito & 2001 & $\begin{array}{l}\text { Empleada de hogar externa que cuida a } \\
\text { una persona dependiente. }\end{array}$ & $\begin{array}{l}\text { Divorciada y casada de nuevo. Tiene seis hijos. } \\
\text { Convive con su marido, cuatro de sus hijas/os y un } \\
\text { nieto. }\end{array}$ \\
\hline 7 & Rocio & 38 & Loja & 2009 & $\begin{array}{l}\text { Médica en una residencia de personas } \\
\text { mayores. }\end{array}$ & $\begin{array}{l}\text { Casada y madre de un hijo que es pequeño. Conviven } \\
\text { los tres. }\end{array}$ \\
\hline 8 & Maribel & 42 & Quito & 1995 & $\begin{array}{l}\text { Trabajadora del Servicio de Ayuda a } \\
\text { Domicilio del Ayuntamiento de Madrid. }\end{array}$ & $\begin{array}{l}\text { Casada, tiene un hijo y una hija que son pequeños. } \\
\text { Conviven los cuatro. }\end{array}$ \\
\hline 9 & Felicia & 46 & Loja & 1994 & $\begin{array}{l}\text { Trabajadora del Servicio de Ayuda a } \\
\text { Domicilio del Ayuntamiento de Madrid. }\end{array}$ & $\begin{array}{l}\text { Divorciada, madres de dos hijos adultos que } \\
\text { conviven con ella. }\end{array}$ \\
\hline 10 & Judith & 47 & Loja & 1993 & $\begin{array}{l}\text { Empleada de hogar externa que cuida a } \\
\text { varias personas dependientes. }\end{array}$ & $\begin{array}{l}\text { Casada, madre de dos hijas, adulta y adolescente. } \\
\text { Conviven los cuatro. }\end{array}$ \\
\hline 11 & Veronica & 57 & Guayaquil & 2000 & $\begin{array}{l}\text { Trabajadora del Servicio de Teleasistencia } \\
\text { del Ayuntamiento de Madrid. }\end{array}$ & $\begin{array}{l}\text { Separada y madre de cuatro hijos e hijas. Convive con } \\
\text { su expareja y con sus hijos/as. }\end{array}$ \\
\hline 12 & Trinidad & 57 & Quito & 2000 & $\begin{array}{l}\text { Empleada de hogar externa que cuida } \\
\text { personas dependientes y también realiza } \\
\text { tareas solamente de limpieza. }\end{array}$ & $\begin{array}{l}\text { Viuda, no tiene hijos. Vive con una pareja de } \\
\text { personas mayores para la que realiza tareas } \\
\text { domésticas a cambio de una habitación (no hay } \\
\text { remuneración monetaria). }\end{array}$ \\
\hline
\end{tabular}




\begin{tabular}{|c|c|c|c|c|c|c|}
\hline 13 & Alba & 58 & Guayaquil & 1994 & $\begin{array}{l}\text { Empleada de hogar externa que cuida a } \\
\text { varias personas dependientes. }\end{array}$ & $\begin{array}{l}\text { Soltera y sin descendencia. Comparte piso con otra } \\
\text { mujer ecuatoriana. }\end{array}$ \\
\hline 14 & Mireia & 39 & Loja & 2007 & $\begin{array}{l}\text { Empleada de hogar interna que cuida a } \\
\text { una persona dependiente. }\end{array}$ & $\begin{array}{l}\text { Soltera y sin descendencia. Vive con la persona que } \\
\text { cuida (interna). }\end{array}$ \\
\hline 15 & Milagros & 45 & $\begin{array}{l}\text { Santa Rosa, } \\
\text { Machala }\end{array}$ & 1996 & $\begin{array}{l}\text { Trabajadora del Servicio de Ayuda a } \\
\text { Domicilio del Ayuntamiento de Madrid. }\end{array}$ & $\begin{array}{l}\text { Casada, madre de un hijo y abuela de un nieto que } \\
\text { están en Ecuador. Convive con su marido. }\end{array}$ \\
\hline 16 & Consuelo & 51 & Loja & 1994 & $\begin{array}{l}\text { Gerocultora en una residencia de personas } \\
\text { mayores (de lunes a viernes) y empleada } \\
\text { de hogar interna con personas } \\
\text { dependientes durante el fin de semana. }\end{array}$ & $\begin{array}{l}\text { Casada y sin descendencia. Convive con su marido. } \\
\text { Los fines de semana ambos trabajan como internos } \\
\text { para una misma pareja de personas mayores. }\end{array}$ \\
\hline 17 & Paulina & 53 & Guayaquil & 2002 & $\begin{array}{l}\text { Empleada de hogar externa que cuida a } \\
\text { varias personas dependientes. }\end{array}$ & Soltera, no tiene descendencia. Convive con su pareja. \\
\hline 18 & Mercedes & 59 & $\begin{array}{l}\text { Chimborazo, } \\
\text { Riobamba }\end{array}$ & 2001 & $\begin{array}{l}\text { Empleada de hogar interna que cuida a } \\
\text { una persona dependiente. }\end{array}$ & $\begin{array}{l}\text { Soltera y sin descendencia. Convive con la persona a } \\
\text { la que cuida (interna) }\end{array}$ \\
\hline 19 & Danira & 55 & Guayaquil & 2001 & $\begin{array}{l}\text { Empleada de hogar externa contratada } \\
\text { para cuidar de una persona dependiente } \\
\text { que está ingresada en un centro para } \\
\text { personas con discapacidad. }\end{array}$ & Soltera, madre de una hija. Comparte piso. \\
\hline 20 & Pilar & 64 & Guayaquil & 2001 & $\begin{array}{l}\text { Trabajadora para una empresa que presta } \\
\text { servicios de ayuda a domicilio sin } \\
\text { concierto público. }\end{array}$ & $\begin{array}{l}\text { Viuda, madre de tres hijas adultas, y abuela. Vive con } \\
\text { su hermana, su hija y su nieta. }\end{array}$ \\
\hline 21 & Leonor & 48 & $\begin{array}{c}\text { Santo } \\
\text { Domingo }\end{array}$ & 2002 & $\begin{array}{l}\text { Empleada de hogar externa que cuida a } \\
\text { varias personas dependientes. }\end{array}$ & $\begin{array}{l}\text { Casada y madre de tres hijos (adultos). Convive con } \\
\text { marido e hijos. }\end{array}$ \\
\hline 22 & Inmaculada & 50 & Guápulo & 2000 & $\begin{array}{l}\text { Gerocultora en residencia de personas } \\
\text { mayores. }\end{array}$ & $\begin{array}{l}\text { Casada y madre de una hija (adulta) y un hijo (niño). } \\
\text { Convive con su marido e hijos/as. }\end{array}$ \\
\hline 23 & Estrella & 68 & Guayaquil & 1994 & $\begin{array}{l}\text { Empleada de hogar interna que cuida a } \\
\text { una persona dependiente. }\end{array}$ & $\begin{array}{l}\text { Separada, tiene una hija y un hijo y un nieto. Es } \\
\text { interna pero cuando sale convive con su nieto } \\
\text { (adulto). }\end{array}$ \\
\hline 24 & Estela & 55 & Quito & 1999 & $\begin{array}{l}\text { Gerocultora en residencia de personas } \\
\text { mayores. }\end{array}$ & $\begin{array}{l}\text { Soltera y sin descendencia. Convive con su hermana } \\
\text { y dos sobrinos/as. }\end{array}$ \\
\hline
\end{tabular}

Atlánticas. Rev. Int. Est. Fem. 2020, 5, 1, 116-145 


\section{BIBLIOGRAFÍA}

Ajenjo, M., García-Román, J. (2019). La persistente desigualdad de género en el uso del tiempo en España. Perspectives Demogràfiques, 14, 1-4. doi: https://doi.org/10.46710/ced.pd.esp.14

Alonso, L. E. (1998). La mirada cualitativa en sociología. Madrid: Fundamentos.

Amelina, A. y Lutz, H. (eds.) (2019). Gender and migration: transnational and intersectional prospects. Abingdon, Oxon: Routledge.

Anderson, B. y Shutes, I. (eds.) (2014). Migration and Care Labour. Theory, Policy and Politics. Londres: Palgrave Macmillan.

Anthias, F. y Yuval-Davis, N. (1983). Contextualizing Feminism: Gender, Ethnic and Class Divisions. Feminist Review, 15, 62-75. doi: https: / / doi.org/10.2307/1394792

Baldassar, L. y Merla, L. (eds.) (2013). Transnational Families, Migration and the Circulation of Care: Understanding Mobility and Absence in Family Life. Abingdon: Routledge.

Bettio, F., Simonazzi, A., Villa, P. (2006). Change in care regimes and female migration: the 'care drain' in the Mediterranean. Journal of European Social Policy, 16(3), 271-285. doi: https:/ / doi.org/10.1177/0958928706065598

Boccagni, P. (2011). Migrants' social protection as a transnational process: public policies and emigrant initiative in the case of Ecuador. International Journal of Social Welfare, 20(3), 318-325. doi: https://doi.org/10.1111/j.1468-2397.2010.00747.x

Blanco, M. y Pacheco, E. (2003). Trabajo y familia desde el enfoque del curso de vida: dos subcohortes de mujeres mexicanas. Papeles de Población, 9(38), 159-193.

Cachón, L. (2009). La España inmigrante: marco discriminatorio, mercado de trabajo y politicas de integración. Barcelona: Anthropos.

Carling, J., Menjívar, C. y Schmalzbauer, L. (2012). Central Themes in the Study of Transnational Parenthood. Journal of Ethnic and Migration Studies, 38(2), 191-217. doi: https: / / doi.org/10.1080/1369183X.2012.646417

Carrasco, C. (2016). Sostenibilidad de la vida y ceguera patriarcal. Una reflexión necesaria. Atlánticas. Revista Internacional de Estudios Feministas, 1(1), 34-57. doi: https:/ / doi.org/10.17979/arief.2016.1.1.1435

Carrasco, C., Borderías, C. y Torns, T. (eds.) (2011). El trabajo de cuidados: historia, teoría y politicas. Madrid: Catarata.

Carrasquer, P. (2013). El redescubrimiento del trabajo de cuidados: algunas reflexiones desde la sociología. Cuadernos de Relaciones Laborales, 31(1), 91-113. doi: https: / / doi.org/10.5209/rev_CRLA.2013.v31.n1.41633 
Clark, R. L., Glick, J. E. y Bures, R. M. (2009). Immigrant Families Over the Life Course: Research Directions and Needs. Journal of Family Issues, 30(6), 852-872. doi: https:/ / doi.org/10.1177/0192513X09332162

Colectivo Ioé (2001). Relatos desde la entraña de los hogares. Voces de inmigrantes en el servicio doméstico. Ofrim Suplementos 8, 37-63.

Colectivo Ioé et Fernández, M. (2010). Encuesta Nacional de Inmigrantes 2007: el mercado de trabajo y las redes sociales de los inmigrantes. Madrid: Ministerio de Trabajo e Inmigración.

Collins, P. H. (1993). Toward a New Vision: Race, Class and Gender as Categories of Analysis and Connection. Race, Sex E Class, 1, 1, 25-45.

Crenshaw, K. (1989). Demarginalizing the intersection of race and sex: A black feminist critique of antidiscrimination doctrine, feminist theory and antiracist politics. University of Chicago Legal Forum, 14, 139-167.

Díaz-Gorfinkiel, M. y Martínez-Buján, R. (2018). Mujeres migrantes y trabajos de cuidados: transformaciones del sector doméstico en España. Panorama Social, 27, 105-118.

Díaz-Gorfinkiel, M. \& Escrivá, A. (2012). Care of older people in migration contexts: local and transnational arrangements between Peru and Spain. Social Politics, 19(1), 129-141.doi: https://doi.org/10.1093/sp/jxr028

Duffy, M. (2007). Doing the Dirty Work: Gender, Race, and Reproductive Labor in Historical Perspective. Gender and Society, 21(3), 313-336. doi: https://doi.org/10.1177/0891243207300764

Elder, G., Kirkpatrick, M. y Crosnoe, R. (2003). The emergence and development of life course theory. En J.T. Mortimer y M. J. Shanahan (eds.) Handbook of the Life Course (pp. 3-19). New York: Springer.

Elder, G. (1991). Lives and social change. En W. Heinz (ed.) Theoretical Advances in Life Course Research. Status Passages and the Life Course, vol. I, Weinheim: Deutscher Studien Verlag.

Ehrenreich, B. y Hochschild, A. (eds.) (2002). Global Women: Nannies, Maids, and Sex Workers in the New Economy. New York: Henry Holt.

Finotelli, C. y LaBarbera, M.C. (2013). When the exception becomes the rule: The Spanish citizenship regime. Migration Letters, 10 (2), 245-253. doi: https:// doi.org/10.33182/ml.v10i2.146

Fischer, B. y Tronto, J. (1990). Toward a feminist theory of care. En E. Abel y M. Nelson (dir.), Circles of Care: Work and Identity in Women's Lives (pp. 36-54). Albany: SUNY Press.

Glenn, E. N. (1992). From Servitude to Service Work: Historical Continuities in the Racial Division of Paid Reproductive Labor. Signs, 18(1), 1-43. doi: https://doi.org/10.1086/494777 
Herrera, G. (2013). Lejos de tus pupilas: Familias transnacionales, cuidados y desigualdad social en Ecuador. Quito: FLACSO Ecuador/ONU Mujeres.

Herrera, G. (2005). Mujeres ecuatorianas en las cadenas globales de cuidado. En G. Herrera, M. C. Carrillo y A. Torres (eds.), La migración ecuatoriana: redes transnacionalismo e identidades (pp. 281-303). Quito: FLACSO Ecuador.

Hochschild, A. R. (2000). Global Care Chains and Emotional Surplus Value. En W. Hutton y A. Giddens (eds.), On The Edge: Living with Global Capitalism (pp. 130-146). London: Jonathan Cape.

Ibañez, Z. y León, M. (2014). Resisting Crisis at What Cost? Migrant Care Workers in Private Households. En B. Anderson y I. Shutes (eds.), Migration and Care Labour: Theory, Policy and Politics (pp. 110-129). London: Palgrave Macmillam.

INE, Instituto Nacional de Estadística (2020). Población (españoles/extranjeros) por País de Nacimiento, sexo y año http: / / www.ine.es

Iglesias, J. (2010). 'A las carreras'. Una nueva precariedad laboral étnica en la región madrileña: el caso de los trabajadores ecuatorianos. Sociología del Trabajo, 69, 21-47.

Kergoat, D. (2000). Division sexuelle du travail et rapports sociaux de sexe. En H. Hirata, F. Laborie, H. Le Doaré y D. Senotier (eds.), Dictionnaire critique du féminisme (pp. 34-44). Paris: PUF.

Leitner, S. (2003). Varieties of familialism: The caring function of the family in comparative perspective. European Societies, 5(4), 353-375. doi: https:// doi.org/10.1080/1461669032000127642

Lutz, H. (2019). An intersectional analysis of transnational care work and transnational families. En A. Amelina y H. Lutz, (eds.), Gender and migration: transnational and intersectional prospects (pp. 59-82). Abingdon, Oxon: Routledge.

Lutz, H. (ed.) (2008). Migration and domestic work; A European Perspective on a Global Theme. Aldershot: Ashgate.

Martínez-Buján, R. (2014). ¡El trabajo doméstico cuenta! Características y transformaciones del servicio doméstico en España. Migraciones, 36, 275305. doi: https: / / doi.org/10.14422/ mig.i36.y2014.002

Moen, P. y DePasquale, N. (2017). Family care work: a policy-relevant research agenda. International Journal of Care and Caring, 1(1), 45-62. doi: https:// doi.org/10.1332/239788217X14866284542346

Moen, P. (2016). Work Over the Gendered Life Course. En M. J. Shanahan, J. T. Mortimer y M. Kirkpatrick (eds.), Handbook of the Life Course, Vol. 2. (pp. 249-275). New York: Springer.

Moen, P. y Roehling, P. (2005). The career mystique: Cracks in the American dream. Boulder: Rowman \& Littlefield. 
Moré, P. (2019). Vieillissement et migration: les salariées immigrées dans le secteur du care en Espagne. Retraite et société, 80, 13-33. doi: https:/ / doi.org/10.3917/ rs1.080.0013

Moré, P. (2018). Los límites del cuidado. Organización de la asistencia a domicilio para personas mayores en Madrid y París. Revista Internacional de Sociología, 76(1): e086. doi: https:/ / doi.org/10.3989/ ris.2018.76.1.16.25

Moré, P. (2017). Migraciones y cuidados a personas mayores en las grandes ciudades. Madrid: Centro de Investigaciones Sociológicas.

Mortimer, J.T. y Moen, P. (2016). The Changing Social Construction of Age and the Life Course: Precarious Identity. En M. J. Shanahan, J. T. Mortimer y M. Kirkpatrick (eds.), Handbook of the Life Course, Vol. 2 (pp. 111-130). New York: Springer.

Oso, L. y Suárez-Grimalt, L. (2017). Migration and Intergenerational Strategies for Social Mobility. Theoretical and Methodological Challenges. Migraciones, 42, 19-41. doi: https:/ / doi.org/10.14422/mig.i42.y2017.002

Oso, L. (2016). Migración ecuatoriana y estrategias familiares de movilidad social: la relación dialéctica entre el capital financiero y emocional. Investigaciones feministas, 7 (1), 221-240. doi: https:/ / doi.org/10.5209/rev_INFE.2016.v7.n1.51739

Parreñas, R. (2005). Children of Global Migration: Transnational families and Gendered Woes. Stanford: Stanford University Press.

Parry, J., Taylor, R., Pettinger, L. y Glucksmann, M. (2005). Confronting the challenges of work today: New horizons and perspectives. The Sociological Review, 53 (2), 1-18. doi: https://doi.org/10.1111/j.1467954X.2005.00569.x

Pérez-Orozco, A. (2014). Del trabajo doméstico al trabajo de cuidados. En C. Carrasco (ed.) Con voz propia: la economía feminista como apuesta teórica y política (pp. 49-74). Madrid: La Oveja Roja.

Pérez-Orozco, A. (2006). Amenaza tormenta. La crisis de los cuidados y la reorganización del sistema económico. Revista de Economía Crítica, 5, 737.

Recio, C., Moreno-Colom, S., Borràs, V. y Torns, T. (2015). La profesionalización del sector de los cuidados. Revista Zerbitzuan, 60, 179193. doi: http: / / dx.doi.org/10.5569/1134-7147.60.12

Rodríguez, V. (ed.) (2012). Inmigración y cuidados de mayores en la comunidad de Madrid. Madrid: BBVA.

Rodríguez-Cabrero, G. et al (2014). Estado de bienestar en España: transformaciones y tendencias de cambio en el marco de la Unión Europea. EN F. Lorenzo Gilsanz (coord.), VII Informe sobre exclusión y desarrollo social en España (pp. 299-394). Madrid: Fundación FOESSA. 
Sassen, S. (2003). Contrageografías de la globalización: La feminización de la supervivencia. Madrid: Traficantes de Sueños.

Skornia, A. (2015). Renegotiating the Care of Children and the Elderly in the Context of Family Migration: Transnational Arrangements and Entangled Inequalities between Peru and Italy. En A. Erdmute y H. Drotbohm (eds.), Anthropological Perspectives on Care: Work, Kinship, and the Life-Course (pp. 43-67). New York: Palgrave Macmillan.

Torns, T. (2008). El trabajo y el cuidado: cuestiones teórico-metodológicas desde la perspectiva de género. Empiria. Revista de Metodología de Ciencias Sociales, 15, 53-73. https:/ / doi.org/10.5944/empiria.15.2008.1199 